\title{
Extra-peritoneal laparoscopic para-aortic lymphadenectomy - A prospective cohort study of 293 patients with endometrial cancer
}

\author{
Sean C. Dowdy *, Giovanni Aletti, William A. Cliby, Karl C. Podratz, Andrea Mariani \\ Division of Gynecologic Surgery, Mayo Clinic, Rochester, Minnesota 55905, USA
}

Received 25 April 2008

Available online 2 October 2008

\begin{abstract}
Objective. To determine if extra-peritoneal laparoscopic para-aortic (PA) lymphadenectomy allows a reliable assessment of PA nodes in patients with endometrial cancer (EC).

Methods. In October of 2005, a single surgeon began performing extra-peritoneal laparoscopic PA lymphadenectomy for patients with EC. A prospective cohort study was initiated from October 2005 through October 2007. Staging of Group A included extra-peritoneal laparoscopic PA lymphadenectomy, while Group B underwent staging via laparotomy.

Results. In a 24 month period, 293 patients underwent surgical treatment for EC, 203 of them underwent complete staging as determined by previously published criteria. Extra-peritoneal laparoscopic PA lymphadenectomy to the renal veins was successful in 35/38 patients (92\%). Mean BMI was 33.0 for Group A and 32.3 for Group B $(p=$ NS). Mean EBL and hospital stay were lower in Group A compared to Group B (163 vs $373 \mathrm{~cm}^{3}, p<0.0001$; median 2 vs 4 nights, $\left.p<0.001\right)$. The total number of PA nodes harvested was not statistically different between Groups A and B (16.5 vs 19.6). Interestingly, in Group A the total number of PA nodes was greater for patients with BMI>35, (21.6 vs 13.1), while in Group B fewer nodes were removed in obese patients (17.8 vs 20.5).

Conclusions. Extra-peritoneal laparoscopy is a reliable method to routinely reach the level of the renal veins, even in obese patients. This approach was feasible in over $90 \%$ of unselected patients and well-tolerated.
\end{abstract}

(C) 2008 Elsevier Inc. All rights reserved.

Keywords: Endometrial carcinoma; Laparoscopy; Lymphadenectomy

\section{Introduction}

We believe that adequate assessment of lymphatic spread in patients with endometrial cancer requires resection of all nodal tissue at risk. This includes assessment of para-aortic (PA) lymph nodes above the inferior mesenteric artery (IMA) up to the level of the renal veins. In support of this, a recent prospective investigation demonstrated that $77 \%$ of patients with PA metastases harbor disease above the IMA [1]. Furthermore, in $60 \%$ of these patients ipsilateral PA nodes below the IMA were free of disease. Thorough staging results in accurate documentation of disease spread, may act as a therapeutic intervention [2-4], and helps determine the need

\footnotetext{
* Corresponding author. 200 1st Street NW, Rochester, MN 55905, USA. Fax: +15072669300 .

E-mail address: dowdy.sean@mayo.edu (S.C. Dowdy).
}

for and extent of post-operative radiation therapy. Documentation of the status of the PA nodes is particularly important given that $47 \%$ of patients with metastatic pelvic nodes will either have positive PA nodes or will subsequently recur in the PA area [5]. With the exception of patients at risk for vaginal recurrence who receive vaginal brachytherapy alone, it is not our practice to prescribe adjuvant radiotherapy for patients with negative lymph nodes [6]. Given the key role of systematic lymphadenectomy in our treatment algorithm, it was paramount that the completeness of lymph node assessment not be compromised when laparoscopic lymphadenectomy was introduced into our practice.

The adequacy of laparoscopic pelvic lymphadenectomy has been well-documented [7-9]. Laparoscopic PA lymphadenectomy to the level of the IMA is also successful in most patients. In contrast, routine extirpation of all PA lymph nodes to the level of the renal veins may be exceedingly difficult 
laparoscopically, particularly in obese patients [10]. In a trial performed by the GOG, $23 \%$ of patients with endometrial cancer randomized to laparoscopy required laparotomy in order to complete the staging [11]. Reasons for conversion included poor exposure [12]. We found this observation problematic given the high frequency of obesity in patients with endometrial cancer together with our recent data demonstrating that stopping the dissection at the level of the IMA will routinely miss 38$46 \%$ of patients with metastatic PA nodes [1]. Consistent with this observation, there is at least one report of a PA recurrence above the IMA following a laparoscopic infra-mesenteric PA dissection [13].

After evaluation of various techniques, we hypothesized that the extra-peritoneal approach would offer the greatest consistency in lymphatic assessment regardless of patient BMI, and it was this approach that was adopted [14-22]. This technique has been utilized previously for patients with cervical carcinoma to minimize the development of adhesions and potentially reduce the risk of radiation-related complications [23,24]. Since the entire dissection is limited to the retroperitoneum, an additional advantage of this approach is that difficulties with exposure resulting from overlying small bowel loops or obesity are reduced. Here we prospectively assess the first two years of our experience with extra-peritoneal laparoscopic para-aortic lymphadenectomy, specifically examining the thoroughness of the nodal dissection with respect to the IMA in comparison to patients who underwent staging via laparotomy. The surgical technique is also described.

\section{Patients and methods}

In October of 2005, laparoscopic pelvic and para-aortic lymphadenectomy was incorporated into the surgical management algorithm of endometrial carcinoma at Mayo Clinic, Rochester, MN. This included routine use of the extraperitoneal approach for laparoscopic para-aortic lymphadenect- omy. Corresponding with this practice change, demographic, intra-operative, and post-operative data from all patients with primary endometrial carcinoma who were surgically managed from October 2005 to October 2007 were collected in a prospective fashion. All patients were operated on by a gynecologic oncologist, with a single surgeon (SCD) performing all laparoscopic procedures.

As previously reported we use objective criteria to determine the need for lymphadenectomy at our institution and approximately $27 \%$ of patients will not require lymphadenectomy [25]. Thus, patients were either treated with hysterectomy alone (vaginal or abdominal), hysterectomy (vaginal or laparoscopic) and laparoscopic pelvic and para-aortic lymphadenectomy, or hysterectomy and open pelvic and para-aortic lymphadenectomy. Given savings in cost, operating times, and low morbidity, we prefer the transvaginal route if feasible rather than the laparoscopic-assisted vaginal technique for the $27 \%$ of patients who require hysterectomy only.

For patients whose tumor characteristics warrant assessment of lymph nodes, a systematic pelvic and para-aortic lymphadenectomy (not sampling) was routinely performed. Details of the pelvic and para-aortic lymphadenectomy via laparotomy have been previously described [1]. Briefly, pelvic lymphadenectomy consisted of complete skeletonization of the common, external, and internal iliac vessels and harvesting of all fatty and lymphatic tissue above and below the obturator nerve. After mobilization of the ascending and descending colon, all lymphatic tissue was harvested from the lateral, anterior, and medial aspects of the vena cava and aorta to the level of the renal veins. For the purposes of this investigation and an ongoing outcomes analysis, our current surgical algorithm includes histologic assessment of para-aortic lymph nodes according to their position relative to the level of the inferior mesenteric artery (IMA). Thus, tissue removed between the renal vein and the IMA is identified separately from tissue removed between the IMA and aortic bifurcation.

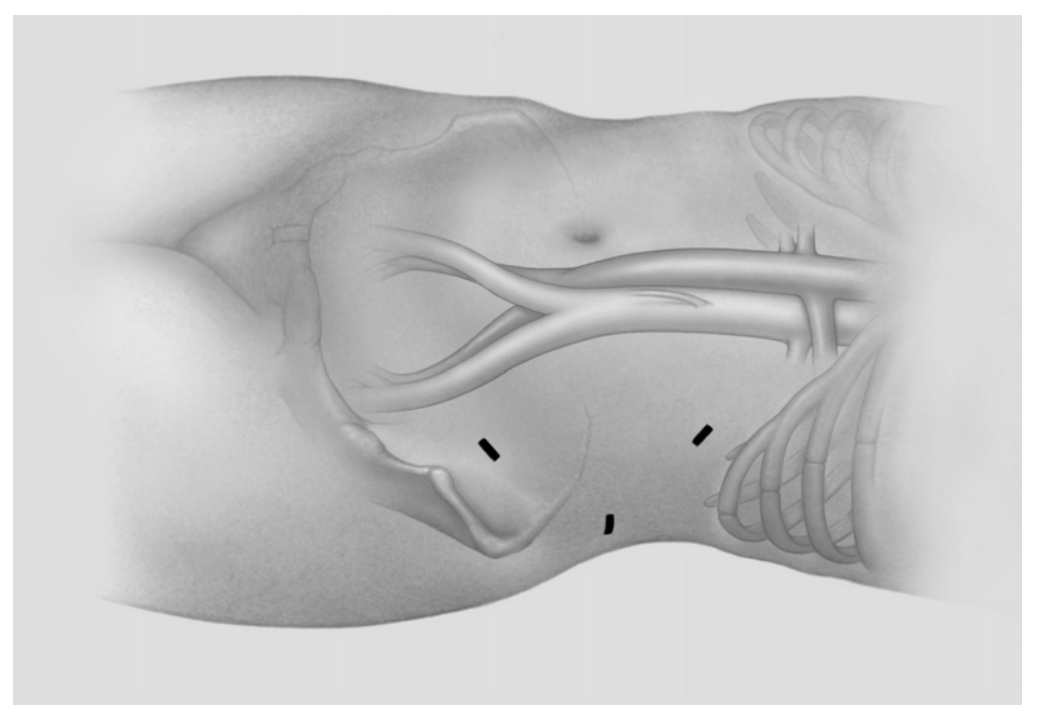

Fig. 1. Schematic illustrating port placement in the left flank. The lowermost port $(10 \mathrm{~mm})$ is located two fingerbreadths medial to, and three to six fingerbreadths superior to, the left anterior superior iliac spine. The remaining trocars are triangulated one finger's length from the initial incision. The second, more posterior trocar is $10 \mathrm{~mm}$. The third, most cephalad $5 \mathrm{~mm}$ trocar is located just inferior to the costal margin. 
The extra-peritoneal dissection was first preceded by a transperitoneal inspection of the abdomen to search for metastatic disease through an umbilical port. In the absence of extrauterine disease a $2 \mathrm{~cm}$ incision was made two fingerbreadths medial to, and three to six fingerbreadths superior to, the left anterior superior iliac spine (Fig. 1). The fibers of the underlying obliques and transversalis muscles were then split until the peritoneum itself was identified. The retroperitoneal space beneath the transversalis muscle was developed posteriorly until the left psoas muscle was palpated. A $10-\mathrm{mm}$ trocar was then positioned in the left flank and the retroperitoneal space insufflated. Additional blunt dissection was then performed utilizing the index finger in the first incision and the laparoscope within the port until the psoas muscle was readily visualized. A 5-mm trocar was then placed under direct visualization and a third trocar placed in the initial incision. Proper placement of these three ports is crucial. Improper placement will handicap the ability of the surgeon to operate in this relatively tight area, or else result in perforation of the peritoneum, making this approach difficult or impossible. Initial insufflation pressures $(10 \mathrm{~mm} \mathrm{Hg})$ and flow rates $(3 \mathrm{~L} / \mathrm{min})$ are low in order to minimize the risk of peritoneal perforation, and theoretically reduce the risk of pneumothorax and hypercarbia. If additional exposure is necessary the pressure can be gradually increased, although $15 \mathrm{~mm} \mathrm{Hg}$ is rarely necessary. Emphysema is commonly noted in the mesentery of the sigmoid colon. However, when the lowermost left flank trocar is converted to an intraperitoneal position for transperitoneal pelvic lymphadenectomy, this emphysema rapidly dissipates and does not interfere with subsequent intraperitoneal procedures.

The left ureter and adjacent gonadal vessels are readily identified just medial to the psoas muscle and allowed to retract anteriorly and out of the field by the pressure of insufflation. The dissection is continued medially until the left common iliac artery and aorta are identified. The gonadal vein is followed superiorly into the left renal vein. At this point all critical anatomy has been identified and the para-aortic nodes between the aortic bifurcation and left renal vein are removed. To remove the right para-aortic nodes, the dissection is continued medially over the aorta and down to the inferior vena cava (IVC). The space between the IVC and lymph nodes is developed on each side of the preserved IMA allowing the nodes to retract anteriorly with the pressure of insufflation in a similar manner as the left ureter. Although not necessary, the right ureter can be identified just lateral to the dissection. Once isolated from the underlying IVC, the right paraaortic nodes are then stripped from the anterior peritoneum, or "roof" of the dissection. After the nodes are removed, the lowermost trocar may be converted to an intraperitoneal position (achieved simply by advancing this trocar through the peritoneum) to be used for the transperitoneal pelvic lymphadenectomy. Thus, a total of 6 trocar incisions are used: three in the left flank (for para-aortic lymphadenectomy, the lowermost left flank trocar is also used for pelvic lymphadenectomy), one in the umbilicus, one in the suprapubic position, and one in the right lower quadrant (for pelvic lymphadenectomy). Operating times for laparoscopic para-aortic lymphadenectomy were determined from video recordings made from each procedure.
Statistical analyses were performed with Fisher's exact tests to evaluate the relationship between pairs of categorical variables. Mann-Whitney $U$ test was used to test for differences between groups in the distributions of continuous measures. Differences between groups were considered statistically significant at $p<0.05$. JMP statistical program (version 4.0.4, SAS Institute, Inc., Cray, North Carolina) was used for the analysis. This investigation was approved by the Institutional Review Board of Mayo Foundation. In accordance with the Minnesota Statute for Use of Medical Information in Research, only those patients who consented to the use of their medical records were included in this analysis.

\section{Results}

In order to reveal any potential selection or referral biases, two comparisons were made between four cohorts of patients. The first two cohorts discussed initially include all patients referred to the surgeon performing laparoscopy (potential candidates for laparoscopic staging) vs all other patients. Succeeding paragraphs consider only those patients in whom extra-peritoneal laparoscopic para-aortic lymphadenectomy was performed (Group A) vs those patients who underwent para-aortic lymphadenectomy via laparotomy (Group B).

In a 24 month period, 293 patients underwent primary surgical treatment for endometrial cancer. Fifty-two patients were referred to the surgeon performing laparoscopic staging and were all initially considered as potential candidates for laparoscopic surgery. The remaining 241 patients were counseled regarding staging by laparotomy, if required, according to protocol. Baseline patient characteristics are shown in Table 1. The laparotomy group included a smaller proportion of patients with stage IC and a larger proportion of

Table 1

Characteristics of patients referred to the surgeon performing laparoscopy vs all others

\begin{tabular}{llcl}
\hline Characteristic & $\begin{array}{l}\text { Potential scope } \\
n(\%)\end{array}$ & $\begin{array}{l}\text { Laparotomy } \\
n(\%)\end{array}$ & $\begin{array}{l}p \\
\text { value }\end{array}$ \\
\hline Age (mean) & 68 & 64.4 & NS \\
BMI (mean) & 33.3 & 33.0 & NS \\
Tumor grade & & & \\
$1 / 2$ & $26(65)$ & $98(60)$ & NS \\
3 & $14(35)$ & $64(40)$ & NS \\
Stage & & & \\
IA & $7(13)$ & $44(18)$ & NS \\
IB & $26(50)$ & $120(50)$ & NS \\
IC & $11(21)$ & $20(8)$ & 0.01 \\
II & $3(6)$ & $2(1)$ & NS \\
IIIA & $1(2)$ & $12(5)$ & NS \\
IIIC & $4(8)$ & $24(10)$ & NS \\
IV & - & $19(8)$ & 0.04 \\
Histological subtype & & & \\
Endometrioid & $44(85)$ & $43(76)$ & NS \\
UPSC & $7(13)$ & $7(30)$ & NS \\
Clear cell & $1(2)$ & $1(0.5)$ & NS \\
MMMT & - & $1(0.5)$ & NS \\
Neuroendocrine & - & & \\
\hline
\end{tabular}

BMI, body mass index; NS, not statistically significant; UPSC, uterine papillary serous carcinoma; MMT, malignant mixed müllerian tumor. 
patients with stage IV disease. Otherwise, no differences were noted with respect to age, BMI, histology, or proportion with positive nodes (stage IIIC).

Staging lymphadenectomy was performed in 203 patients as determined by previously published criteria. For the cohort referred to the surgeon performing laparoscopy, 41 (79\%) were staged compared to $162(67 \%)$ in the laparotomy group. Excluding the four patients referred for laparoscopic staging after hysterectomy elsewhere, $71 \%$ in the former cohort were staged $(p=\mathrm{NS})$.

Of the 52 patients considered for laparoscopic lymphadenectomy, in most cases hysterectomy was performed vaginally without the need for laparoscopic assistance. In the event that the tumor characteristics warranted lymphatic assessment, the patient was then repositioned for laparoscopic staging. Ten patients did not meet criteria for staging and underwent hysterectomy only ( 9 vaginal, 1 abdominal). Complete staging was performed in 41 patients, 35 via extra-peritoneal laparoscopy, 1 via transperitoneal laparoscopy, and 5 via laparotomy. Protocol was breached in $1(2 \%) 85$ year old patient with multiple co-morbidities and only $3 \mathrm{~mm}$ invasion who underwent vaginal hysterectomy, bilateral salpingo-oophorectomy, and laparoscopic pelvic lymphadenectomy only. Thus, $6(12 \%)$ patients had an open procedure, four of which were planned (discussed further below). Extra-peritoneal PA dissection was unsuccessful in three patients because of perforation of the peritoneum: 2 were performed via laparotomy (BMI 51.5 and 37 ) and 1 via transperitoneal laparoscopy. Thus the extraperitoneal approach was successful in 35 of 38 patients (92\%) requiring staging by protocol. The 4 patients who underwent a planned open procedure are described in Table 2. In three of these four patients, laparotomy was planned because the uterus was felt to be too large to be removed without morcellation and/ or there was a suspicion of advanced disease. In a fourth patient the laparoscopic approach was considered unwise because of multiple previous surgeries, including a renal transplant.

Remaining analyses will be limited to patients who underwent lymphadenectomy. Group A includes the 35 patients who underwent laparoscopic extra-peritoneal para-aortic lymphadenectomy, Group B the 167 patients who underwent lymphadenectomy via laparotomy (the single transperitoneal laparoscopic PA lymphadenectomy was eliminated from analysis). Mean BMI was 33.0 (range 21.6-51.6) and 32.3 (range 16.4-58) for Groups A and B, respectively ( $p=\mathrm{NS}$ ).
As described above, in three patients the extra-peritoneal approach was unsuccessful after the peritoneum was perforated during port placement (1 managed with transperitoneal paraaortic lymphadenectomy, the other two via laparotomy). No conversions were performed for hemorrhage and no other intraoperative complications occurred which were related to the extra-peritoneal para-aortic lymphadenectomy. Mean EBL for the entire procedure (hysterectomy and lymphadenectomy) was $163 \mathrm{~cm}^{3}$ for the laparoscopic group compared to $373 \mathrm{~cm}^{3}$ for Group B $(p<0.0001)$. Only 1 patient in Group A was transfused, but her hemoglobin increased from 7.6 to 11.8 after receiving 2 units of packed red blood cells in the recovery room. It may be that the pre-transfusion value was obtained from a blood draw proximal to intravenous administration of crystalloid.

There were 3 (6\%) post-operative complications in Group A. A 78 year old developed post-operative atrial fibrillation resulting in an 8 day admission. She was later readmitted after being over-anticoagulated and noted to have a retroperitoneal hematoma managed with 2 units of packed red blood cells. The post-operative course of a 93 year old was complicated by delirium which required a 6 day hospitalization. Ischemic brain injury was ruled out and the delirium resolved prior to discharge. A third patient underwent a concurrent sling placement for urinary incontinence. She developed a urinary tract infection and adynamic ileus and was hospitalized for 5 days. Although imaging was not performed specifically to search for lymphocysts in the PA area, if present, none were symptomatic.

Median operating time for the extra-peritoneal laparoscopic para-aortic lymphadenectomy was $69 \mathrm{~min}$ (range 20-121). The 20 minute case involved a patient with an extensive anomalous network of left renal vessels which resulted in an abbreviated dissection. The next fastest operating time was $48 \mathrm{~min}$. Operating times were noted to improve with additional experience. The first 10 cases lasted a median of $84.8 \mathrm{~min}$ vs $66.8 \mathrm{~min}$ for the remaining 25 cases $(p=0.03)$. Operating times for para-aortic lymphadenectomy via laparotomy were not recorded separately from the overall operating time prohibiting a direct comparison between groups. There was no correlation between BMI and operating times in the laparoscopic group.

As shown in Table 3, the total number of para-aortic lymph nodes harvested was not statistically different between groups. Furthermore, no differences in the number of lymph nodes were noted when stratified with respect to location above or below

Table 2

Characteristics of patients who were considered for laparoscopy, but underwent planned laparotomy for endometrial cancer

\begin{tabular}{|c|c|c|c|c|c|c|}
\hline Patient & BMI & Procedure & $\begin{array}{l}\text { Uterine weight } \\
\text { (g) }\end{array}$ & Stage & Histology & Other \\
\hline 1 & 58 & TAH, BSO, PPALND, panniculectomy, hernia repair & 885 & $\mathrm{IC}$ & Endometrioid & Huge ventral hernia \\
\hline 2 & 30 & Radical hysterectomy, BSO, PPALND & 720 & IIIC & MMMT & Suspicion of cervical involvement (confirmed) \\
\hline 3 & 40 & Radical hysterectomy, BSO, PPALND, omentectomy & 335 & IIB & UPSC & Suspicion of cervical involvement (confirmed) \\
\hline $4^{\mathrm{a}}$ & 48 & $\mathrm{TAH}, \mathrm{BSO}$ & 230 & IB & Endometrioid & Previous renal transplant \\
\hline
\end{tabular}

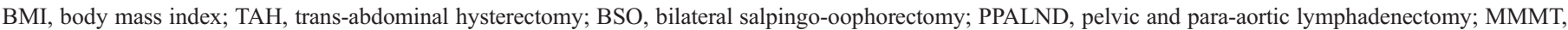
malignant mixed müllerian tumor; UPSC, uterine papillary serous carcinoma.

a Tumor characteristics did not meet criteria for lymphadenectomy. 
Table 3

Para-aortic node counts in patients staged via extra-peritoneal laparoscopy vs laparotomy, stratified by BMI and position relative to the IMA

\begin{tabular}{ccc}
\hline & $\begin{array}{l}\text { Extra-peritoneal } \\
\text { laparoscopy }\end{array}$ & Laparotomy \\
\hline Total nodes & 16.5 & 19.6 \\
BMI $<35$ & $13.1^{* \wedge}$ & $20.5^{\wedge}$ \\
BMI $\geq 35$ & $21.6^{*}$ & 17.8 \\
Above IMA & 9.5 & 11.3 \\
BMI $<35$ & $7.4^{* \wedge}$ & $12.2^{\wedge}$ \\
BMI $\geq 35$ & $12.3^{*}$ & $9.7^{\#}$ \\
Below IMA & 7.9 & 8.5 \\
BMI $<35$ & $6.2^{* \wedge}$ & $8.9^{\wedge}$ \\
BMI $\geq 35$ & $10.2^{*}$ & 7.8 \\
\hline
\end{tabular}

$*, \wedge$, and \# indicate pairs within each subset that are statistically different.

the IMA ( $p=\mathrm{NS})$. However, differences were apparent when the data was stratified by BMI $>35$. Laparotomy resulted in higher para-aortic node counts than laparoscopy for non-obese patients, while the opposite was true for obese patients, although this trend was not statistically significant (Table 3). A trend of improving node counts with additional experience could not be demonstrated.

Median hospital stay was 2 nights for those in Group A (staged laparoscopically) vs 4 nights for those in Group B $(p<0.001)$. For patients not requiring staging, median hospital stay was 3 nights for those who underwent trans-abdominal hysterectomy and bilateral salpingo-oophorectomy vs 2 nights for those who underwent vaginal hysterectomy and bilateral salpingo-oophorectomy only. For Group A, age $>70$ was associated with longer hospital stay, mean 3.7 vs 2.1 nights $(p<0.001)$. Similarly, for the 5 patients who were dismissed within $24 \mathrm{~h}$, their mean age was 63 vs 77 for the 8 patients with $5-8$ day admissions $(p=0.01)$. For Group $\mathrm{B}$, age had no effect on hospital stay (5.3 vs 5.6 nights). BMI had no impact on hospital stay for Group A, while this was of borderline significance in Group B (5.2 vs 6.5 nights for $\mathrm{BMI}>35$, $p=0.059)$.

Longest follow-up was 2 years, prohibiting a valid survival analysis. However, only 1 patient from the laparoscopic cohort has recurred, a patient with a grade 3, stage IIIA tumor with full thickness myometrial invasion. Post-operatively she was treated with pelvic radiation. Chemotherapy with IV carboplatin and paclitaxel was discontinued after she developed neutropenic sepsis. Her isolated central recurrence occurred 12 months after primary surgery and was managed with posterior exenteration at the time of preparation of this manuscript. She is scheduled to receive single agent carboplatin at a dose reduction.

\section{Discussion}

Through the pioneering efforts of surgeons in this country and across Europe, the widespread adoption of laparoscopic surgery represents perhaps one of the most significant changes in the surgical aspect of gynecologic oncology. However, the utilization of laparoscopy for patients with endometrial cancer is accompanied by unique challenges given its known association with obesity. While laparoscopic surgery in obese patients can be time consuming and extremely difficult, we must accept that most patients with endometrial cancer are obese, and we are therefore obliged to devise techniques to provide obese patients with the same standards of care as patients who are thin.

Previous publications investigating the use of laparoscopic transperitoneal para-aortic lymphadenectomy for patients with endometrial cancer have identified potential shortcomings of this approach. For example, in a review of 206 patients with a BMI $>35$ with endometrial cancer, the median number of paraaortic nodes removed in the transperitoneal laparoscopic cohort was only 6 [10]. Such a result from an institution known for their surgical expertise suggests an inherent weakness not in the surgeon, but in the procedure itself. Perhaps because of these very constraints, there is evidence that utilization of transperitoneal laparoscopy for endometrial cancer staging is limited outside of tertiary care institutions. In a review of over 12,000 patients from two California databases, Leiserowitz, et al. demonstrated that patients who underwent laparoscopic procedures for endometrial cancer were younger and had significantly fewer comorbid conditions than did those who underwent laparotomy [26]. Patients who underwent laparoscopy were also much less likely to be obese. Thus, the use of transperitoneal laparoscopic staging appears to be limited to the most favorable operative candidates at the present time. It is unfortunate that the benefits of minimally invasive surgery cannot be more consistently applied to the cohort of patients with the most potential to benefit.

In the present study we failed to demonstrate any differences in patient demographics, including BMI, between groups. All patients with endometrial cancer referred to the laparoscopic surgeon were reported and only 4 patients $(8 \%)$ underwent a planned open procedure, all for valid reasons (Table 2). Despite this, there were no differences in paraaortic lymph node counts between groups. Although paraaortic node counts were somewhat higher in the laparoscopic cohort for patients with $\mathrm{BMI}>35$, this finding is likely to be spurious due to the relatively small size of the laparoscopic cohort. Alternatively, lymphadenectomy via laparotomy was superior for patients with $\mathrm{BMI}<35$, but these differences do not appear to be clinically relevant given that assessments by both approaches were quite thorough. Overall, these data support the conclusion that the laparoscopic extra-peritoneal para-aortic approach is at least equivalent to assessment via laparotomy in the average, and particularly in the obese, patient. This is also indirectly supported by a lack of a correlation between operative times and BMI. That PA lymphadenectomy can be performed safely and thoroughly, even in the most obese patients using this technique is noteworthy. In the most obese patient of this series to undergo laparoscopic PA lymphadenectomy (BMI 51.6), 34 para-aortic nodes were harvested, 21 of which were located above the IMA.

We demonstrate that the laparoscopic extra-peritoneal approach is successful in $92 \%$ of patients. No patient was considered ineligible for laparoscopy based on weight or comorbid conditions alone. In experienced hands this 
proportion may well be higher as this series includes the first operations to be performed by this surgeon. A similarly high success rate has also been demonstrated by other groups in both endometrial and cervical cancer $[17,18]$. Infrequent complications were minor and not specific to the technique described here. Significant improvements in estimated blood loss and hospital stay compared to the laparotomy group were also observed. These findings are consistent with those described by the GOG in their randomized trial [11]. Thus, the advantages demonstrated for transperitoneal laparoscopy appear to apply also to the extra-peritoneal approach. The maturity of our data is at present not adequate to perform an outcomes comparison.

The extra-peritoneal approach may offer more advantages than just exposure in obese patients. Lowe, et al. demonstrated the feasibility of teaching this technique to fellows [27]. Another important advantage is that in contrast to transperitoneal para-aortic lymphadenectomy, extra-peritoneal laparoscopy requires no expert assistance. Only three ports are used, such that the first assistance is required only to direct the optic.

A disadvantage of extra-peritoneal laparoscopy is that it is not widely utilized in the United States and instruction is currently limited. The reluctance to utilize this procedure may be due to a lack of familiarity with the extra-peritoneal approach itself. Surgeons with past experience in extra-peritoneal laparotomy to remove para-aortic nodes from patients with advanced cervical cancer may be confident enough to attempt this technique without specific training. An additional disadvantage is that the integrity of the peritoneum must be preserved. In the event that the peritoneum is perforated, the advantage provided by the extra-peritoneal pneumoperitoneum in terms of exposure and retraction is lost. It is possible, however, to tamponade small peritoneal defects using a Foley catheter balloon, if they do occur. The Foley catheter is advanced directly through the skin and peritoneal defect after removing the port. This is facilitated by transperitoneal visualization using an umbilical port. The catheter is then inflated, placed on traction, and the port replaced immediately adjacent to the Foley.

This investigation was not designed to directly compare transperitoneal and extra-peritoneal laparoscopy and is not intended to suggest superiority of one approach over another. But our data do suggest that extra-peritoneal laparoscopy may have advantages, particularly in obese patients, given the ease of reaching the renal veins. While we believe that dissection up to the renal veins is prudent and necessary in patients that require lymphadenectomy, this investigation was not intended to support this concept.

Although this investigation was prospective and included a relatively large number of patients, several shortcomings and potential biases exist. All laparoscopic procedures were performed by a single surgeon and these results must be replicated by others before they may be extrapolated to other practices. However, it should be pointed out that these data represent the very first patients to undergo laparoscopic lymphadenectomy by this surgeon, supporting the notion that these results should easily be attained by others. Although the total number of patients was relatively large, only 35 patients underwent extra-peritoneal para-aortic lymphadenectomy, suggesting expansion of this cohort followed by further analysis to confirm our present findings.

This investigation was non-randomized. While referral bias is a potential source of confounding, this effect should be relatively minor in our practice. Referrals to our division are handled by a central appointment desk that makes assignments according to availability rather than seniority to ensure equitable distribution of cases and patient access. Furthermore, analysis of patient and tumor characteristics showed no significant differences between groups with regard to age, histologic subtype, or BMI. Stage distribution was also very similar, although the laparoscopic group had a higher proportion with stage IC disease, and a lower proportion with stage IV disease. This finding may be sporadic given the small number of patients in these groups.

In conclusion, extra-peritoneal laparoscopy is an easily learned technique which may have advantages over transperitoneal laparoscopy for assessment of the para-aortic lymph nodes in patients with endometrial cancer. These advantages appear to be particularly apparent in patients with BMI $>35$. Average operating time was just over one hour, and was not correlated with BMI. No intra-operative complications occurred in this series. Extra-peritoneal laparoscopy has the same benefits as traditional laparoscopy in regards to blood loss and post-operative recovery. For gynecologic oncologists who believe in the importance of complete para-aortic assessment, the extra-peritoneal approach is a feasible option.

\section{Conflict of interest statement}

The authors have no conflicts of interest to declare.

\section{Acknowledgments}

The corresponding author wishes to express his sincere gratitude to Dr. Eric Leblanc at Oscar Lambre Cancer Center in Lille, France, Prof. Dr. Achim Schneider, and Dr. Christhardt Köhler at Charite Hospital, Berlin, Germany, for their expertise and willingness to instruct a novice in the technique of laparoscopy. Their hospitality, generosity, and kindness are indicative of an underlying altruism that transcends cultural boundaries and truly embodies the spirit of Dr. William Mayo's aphorism, "The best interest of the patient is the only interest to be considered."

\section{References}

[1] Mariani A, Dowdy SC, Cliby WA, Gostout BS, Jones MB, Wilson TO, Podratz KC. Prospective assessment of lymphatic dissemination in endometrial cancer: a paradigm shift in surgical staging. Gynecol Oncol 2008; 109:11-8.

[2] Cragun JM, Havrilesky LJ, Calingaert B, Synan I, Secord AA, Soper JT, Clarke-Pearson DL, et al. Retrospective analysis of selective lymphadenectomy in apparent early-stage endometrial cancer. J Clin Oncol 2005;23: $1675-3668$.

[3] Kilgore LC, Partridge EE, Alvarez RD, Austin JM, Shingleton HM, Noojin Fr, Conner W. Adenocarcinoma of the endometrium: survival comparisons of patients with and without pelvic node sampling. Gynecol Oncol 1995;56:1995. 
[4] Mariani A, Webb MJ, Galli L, Podratz KC. Potential therapeutic role of paraaortic lymphadenectomy in node-positive endometrial cancer. Gynecol Oncol 2000;76:348-56.

[5] Mariani A, Keeney GL, Aletti G, Webb MJ, Haddock MG, Podratz KC. Endometrial carcinoma: paraaortic dissemination. Gynecol Oncol 2004; 92:833-8

[6] Mariani A, Dowdy SC, Keeney GL, Long HJ, Lesnick TG, Podratz KC. High-risk endometrial cancer subgroups: candidates for target-based adjuvant therapy. Gynecol Oncol 2004;95:120-6.

[7] Childers JM, Hatch K, Surwit EA. The role of laparoscopic lymphadenectomy in the management of cervical carcinoma. Gynecol Oncol 1992;47:38-43.

[8] Childers JM, Spirtos NM, Brainard P, Surwit EA. Laparoscopic staging of the patient with incompletely staged early adenocarcinoma of the endometrium. Obstet Gynecol 1994;83:597-600.

[9] Kohler C, Klemm P, Schau A, Possover M, Krause N, Tozzi R, Schneider A. Introduction of transperitoneal lymphadenectomy in a gynecologic oncology center: analysis of 650 laparoscopic pelvic and/or paraaortic transperitoneal lymphadenectomies. Gynecol Oncol 2004;95:52-61.

[10] Eisenhauer EL, Wypych KA, Mehrara BJ, Lawson C, Chi DS, Barakat RR, Abu-Rustum NR. Comparing surgical outcomes in obese women undergoing laparotomy, laparoscopy, or laparotomy with panniculectomy for the staging of uterine malignancy. Ann Surg Oncol 2007;14:2384-91.

[11] Walker JL, Piedmonte M, Spitros N, Eisenkop S, Schlaerth J, Mannel R, Spiegel G. Phase III trial of laparoscopy (scope) vs. laparotomy (open) for surgical resection and comprehensive surgical staging of uterine cancer: a Gynecologic Oncology Group (GOG) study funded by NCI. Gynecol Oncol 2006;101:S2.

[12] Kornblith A, Walker JL, Huang H, Cella D. Quality of Life (QOL) of patients in a randomized clinical trial of laparoscopy (scope) vs. open laparotomy (open) for the surgical resection and staging of uterine cancer: a Gynecolgic Oncology Group (GOG) study. Gynecol Oncol 2006;101:S22.

[13] Eitan R, Abu-Rustum NR, Walker JL, Barakat RR. Endometrial cancer metastatic to infrarenal aortic lymph nodes unrecognized during laparoscopic inframesenteric aortic lymph node dissection. Gynecol Oncol 2004;93:260-2.

[14] Coptcoat MJ. Extraperitoneal pelvic and para-aortic lymphadenectomy. Endosc Surg Allied Technol 1995;3:9-15.

[15] Dargent D, Ansquer Y, Mathevet P. Technical development and results of left extraperitoneal laparoscopic paraaortic lymphadenectomy for cervical cancer. Gynecol Oncol 2000;77:87-92.

[16] LeBlanc E, Caty A, Dargent D, Querleu D, Mazeman E. Extraperitoneal laparoscopic para-aortic lymph node dissection for early stage nonsemi- nomatous germ cell tumors of the testis with introduction of a nerve sparing technique: description and results. J Urol 2001;165:89-92.

[17] Leblanc E, Narducci F, Frumovitz M, Lesoin A, Castelain B, Baranzelli $\mathrm{MC}$, et al. Therapeutic value of pretherapeutic extraperitoneal laparoscopic staging of locally advanced cervical carcinoma. Gynecol Oncol 2007;105: 304-11.

[18] Nagao S, Fujiwara K, Kagawa R, Kozuka Y, Oda T, Maehata K, et al. Feasibility of extraperitoneal laparoscopic para-aortic and common iliac lymphadenectomy. Gynecol Oncol 2006;103:732-5.

[19] Querleu D, Dargent D, Ansquer Y, Leblanc E, Narducci F. Extraperitoneal endosurgical aortic and common iliac dissection in the staging of bulky or advanced cervical carcinomas. Cancer 2000;88:1883-91.

[20] Sanjuan A, Illa M, Torne A, Roman SM, Jurado M, Lejarcegui JA, Pahisa J. Extraperitoneal laparoscopic para-aortic lymphadenectomy as a diagnostic procedure for lymph node recurrence of gynaecological cancers. Acta Obstet Gynecol Scand 2007;86:491-5.

[21] Sonoda Y, Leblanc E, Querleu D, Castelain B, Papageorgiou TH, Lambaudie E, Narducci F. Prospective evaluation of surgical staging of advanced cervical cancer via a laparoscopic extraperitoneal approach. Gynecol Oncol 2003;91:326-31.

[22] Tillmanns T, Lowe MP. Safety, feasibility, and costs of outpatien laparoscopic extraperitoneal aortic nodal dissection for locally advanced cervical carcinoma. Gynecol Oncol 2007;106:370-4.

[23] Lanvin D, Elhage A, Henry B, Leblanc E, Querleu D, Delobelle-Deroide A. Accuracy and safety of laparoscopic lymphadenectomy: an experimental prospective randomized study. Gynecol Oncol 1997;67:83-7.

[24] Occelli B, Narducci F, Lanvin D, Querleu D, Coste E, Castelain B, et al. De novo adhesions with extraperitoneal endosurgical para-aortic lymphadenectomy versus transperitoneal laparoscopic para-aortic lymphadenectomy: a randomized experimental study. Am J Obstet Gynecol 2000;183: 529-33.

[25] Mariani A, Webb MJ, Keeney GL, Haddock MG, Calori G, Podratz KC. Low-risk corpus cancer: is lymphadenectomy or radiotherapy necessary? Am J Obstet Gynecol 2000;182:1506-19.

[26] Leiserowitz GS, Xing GB, Parihk-Patel A, Cress R, Abidi A, Mourton SM, et al. Survival of endometrial cancer patients after laparoscopically assisted vaginal hysterectomy or total abdominal hysterectomy: analysis of risk factors. Gynecol Oncol 2007;104:S5.

[27] Lowe MP, Bahador A, Muderspach LI, Burnett A, Santos L, Caffrey A, et al. Feasibility of laparoscopic extraperitoneal surgical staging for locally advanced cervical carcinoma in a gynecologic oncology fellowship training program. J Minim Invasive Gynecol 2006;13:391-7. 